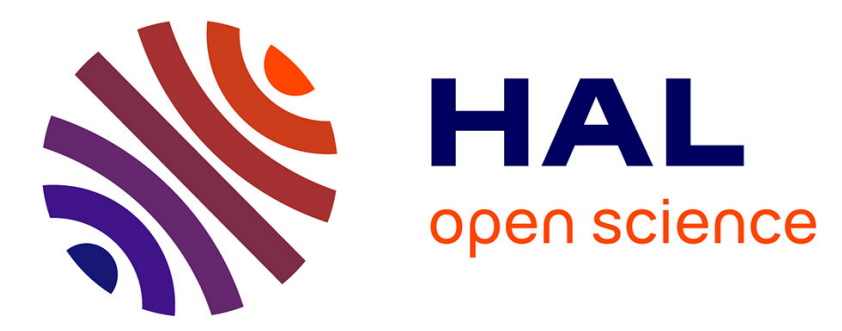

\title{
Modelling of sound scattering by the sea floor
}

A. Ivakin

\section{To cite this version:}

A. Ivakin. Modelling of sound scattering by the sea floor. Journal de Physique IV Proceedings, 1994, 04 (C5), pp.C7-1095-C7-1098. 10.1051/jp4:19945241 . jpa-00252929

\section{HAL Id: jpa-00252929 https://hal.science/jpa-00252929}

Submitted on 1 Jan 1994

HAL is a multi-disciplinary open access archive for the deposit and dissemination of scientific research documents, whether they are published or not. The documents may come from teaching and research institutions in France or abroad, or from public or private research centers.
L'archive ouverte pluridisciplinaire HAL, est destinée au dépôt et à la diffusion de documents scientifiques de niveau recherche, publiés ou non, émanant des établissements d'enseignement et de recherche français ou étrangers, des laboratoires publics ou privés. 


\title{
Modelling of sound scattering by the sea floor
}

\author{
A.N. IVAKIN \\ N.N. Andreev Acoustics Institute, Russian Academy of Sciences, 4 Shvernik Street, Moscow 117036, \\ Russia
}

\begin{abstract}
Geoacoustic model of sound scattering by the sea floor is developed, in which the floor is considered to be irregular layered medium with random scatterers of two types: the roughness of seabed interfaces and the volume inhomogeneities (spatial fluctuations of the acoustical parameters). As an example for computer simulation, backscattering by a silty layer overlaying a sandy half-space has been considered, and the angular dependences of scattering coefficient have been calculated.
\end{abstract}

\section{INTRODUCTION}

Modelling of sound scattering by the sea floor is of practical interest due to different reasons. First, there is practical need for predicting of the bottom reverberation, which critically affects the operation of underwater acoustic systems. Secondly, this modelling is the necessary stage in development of the methods of remote acoustic sensing, which have a great number of applications, such as obtaining information on seabed sediment properties, its relief and internal structure, discovering mineral and biological resourses, monitoring of ecological state of the sea floor and others.

The most of publications on the problem deal with the modelling of highfrequency scattering, where the seabed stratification or layerity is not taken into account. In this case sound scattering by roughness of bottom relief and near surface inhomogeneities has been studied in adequate detail [1-5]. When frequency is lowered, sound penetration into the bottom increases and the influence of the stratification, scattering by the deep interfaces and layers can become essential. In a number of recent papers [5-8], this problem has been considered for some cases and particular bottom models. Here we consider more general model, including both volume and rougness scattering for arbitrary regular bottom stratification.

\section{BOTTOM MODEL}

Let the bottom is randomly inhomogeneous medium, which is plane-layered on the average and contains the irregularities of two types the volume inhomogeneities (spatial fluctuations of the acoustical parameters) and the roughness of interfaces. We define the interfaces in the bottom medium by the random functions $z=z_{j}+\sigma_{j} \zeta_{j}(\vec{r}), \vec{r}=\{x, y\},\left\langle\zeta_{j}\right\rangle=0,\left\langle\zeta_{j}^{2}\right\rangle=1, j=1,2, \ldots$, N. Here the angle brackets $\langle\ldots\rangle$ signify the ensenble averaging, $\sigma_{j}$ is the rms of roughness of 
$j$-interface. For the bottom surface (water-sediment interface), let $j=1$ and $z_{1}=0$.

The local refraction index $\tilde{n}$ and the local relative density $\tilde{m}$ (the relation of sediment and water densities) we define by the random functions $\tilde{n}^{2}=n^{2}(z)(1+$ $\left.\varepsilon(z) \eta_{1}(\vec{R})\right), \quad \tilde{m}=m(z)\left(1+\mu(z) \eta_{2}(\vec{R})\right), \vec{R}=\{\vec{r}, z\},\left\langle\eta_{1}\right\rangle=\left\langle\eta_{2}\right\rangle=0,\left\langle\eta_{1}^{2}\right\rangle=\left\langle\eta_{2}^{2}\right\rangle=1$, where $\mathrm{n}$ and $\mathrm{m}$ are regular (averaged) refraction index and relative density being dependent on the depth $z, \varepsilon$ and $\mu$ are rms of corresponding random spatial fluctuations and are to be defined as the regular depth-functions too.

It is known that the acoustical parameters of sea sediment are determined in the main by its porosity [9]. If the local random fluctuations of the porosity $\tilde{\eta}(\vec{R})$ are sufficiently small, we can use the linear approximations $\eta_{1} \simeq \eta(\vec{R})=\tilde{\eta} /\left\langle\tilde{\eta}^{2}\right\rangle^{1 / 2}$ and $\eta_{2}$ $\simeq-\eta(\vec{R})$. Here the minus is due to the density and sound velocity are usually reduced when the sediment porosity rises.

\section{SCATTERING AMPLITUDE}

Let a plane sound wave of unit amplitude $p$, arrives from the homogeneous water half-space $z<0$ and is incident on the bottom: $p_{i}=\exp \left(i \vec{\alpha}_{0} \vec{r}_{1}+i \nu\left(\alpha_{0}\right) z\right)$, where $\vec{\alpha}_{0}$ and $v_{0}=\left(k^{2}-\alpha_{0}^{2}\right)^{1 / 2}$ are the horizontal and vertical components of the incident wave vector, $k$ is its wave number. The scattered field $p_{s}$ in the water may be represented by an expansion in plane waves:

$p_{s}=\int v^{-1}(\alpha) A\left(\vec{\alpha}, \vec{\alpha}_{0}\right) \exp (i \vec{\alpha} \vec{\Gamma}-i v(\alpha) z) d^{2} \alpha$

where $A$ is the scattering amplitude. When it has been found, all the basic characteristics of the scattered field can be determined. For example, the scattering coefficient $M$, which characterizes the frequency-angular distribution of mean intensity of the scattered field in the Fraunhofer zone (or far zone, relative to a scattering volume), can be determined from the equation

$M=4 \pi^{2}\left\langle\left|A\left(\vec{\alpha}, \vec{\alpha}_{0}\right)\right|^{2}\right\rangle / S$

where $S$ is the horizontal cross section of the scattering volume.

In the first approximation of the method of small perturbations in the small parameters $\varepsilon, \mu$, and $k \sigma$, we obtain the next equations for the scattering amplitude

$$
\begin{aligned}
& A\left(\vec{\alpha}, \vec{\alpha}_{0}\right)=\left(i k^{2} / 2\right)\left(\sum_{j} \sigma_{j} f_{j}\left(\vec{\alpha}_{,}, \vec{\alpha}_{0}\right) \hat{\zeta}_{j}\left(\vec{\alpha}_{-} \vec{\alpha}_{0}\right)+\int f\left(\vec{\alpha}_{,} \vec{\alpha}_{0}, z\right) \hat{\eta}\left(\vec{\alpha}^{-} \vec{\alpha}_{0}, z\right) d z\right) \\
& \hat{\zeta}_{j}(\vec{\kappa})=(2 \pi)^{-2} \int \zeta_{j}(\vec{r}) \exp (-i \vec{k} \vec{r}) d^{2} r \\
& \hat{\eta}_{j}(\vec{\kappa}, z)=(2 \pi)^{-2} \int \eta_{j}(\vec{r}, z) \exp (-i \vec{\kappa} \vec{r}) d^{2} r \\
& f\left(\vec{\alpha}, \vec{\alpha}_{0}, z\right)=\left(\left(n^{2} / m\right)(\varepsilon+\mu)+(\mu / m) \vec{\alpha}_{0} / \vec{\alpha}^{2}\right) \varphi_{1}\left(\alpha, \alpha_{0}, z\right)+\mu m \varphi_{2}\left(\alpha, \alpha_{0}, z\right) \\
& f_{j}\left(\vec{\alpha}, \vec{\alpha}_{0}\right)=\left(\Delta_{j}\left(n^{2} / m\right)+\Delta_{j}(1 / m) \vec{\alpha}_{0} \vec{\alpha}_{0} / k^{2}\right) \varphi_{1}\left(\alpha, \alpha_{0}, z\right)+\Delta_{j}(m) \varphi_{2}\left(\alpha, \alpha_{0}, z\right)
\end{aligned}
$$


where $\Delta_{j}(\ldots)$ is the value of discontinuity of the appropriate parameter at the mean interfaces $z=z_{j}$; for example, $\Delta_{j}(m)=m_{j-1}\left(z_{j}\right)-m_{j}\left(z_{j}\right)$, where $m_{j}(z)$ is the mean relative density in the j-layer; $\varphi_{1}$ and $\varphi_{2}$ are the continuous regular functions $\varphi_{1}=\psi(z, \alpha) \psi\left(z, \alpha_{0}\right)$ and $\varphi_{2}=\mathrm{m}^{-1}(\mathrm{~d} \psi(z, \alpha) / \mathrm{d} z)\left(\mathrm{d} \psi\left(z, \alpha_{0}\right) / \mathrm{d} z\right)$. Here $\psi(z, \alpha)$ is the

function, which describes the acoustic field induced by the incident plane wave of unit amplitude in the regular plane-layered medium with the relative density $m(z)$ and the refraction index $n(z)$, and can be found by means of wel1-developed procedure [10] being used widely for calculation of the plane wave reflection coefficient $V(\alpha)=\psi(0, \alpha)-1$

\section{SCATTERING COEFFICIENT.}

Making use of the formulae $(1,2)$, we obtain the next equations for the scattering coefficient of the bottom

$$
\begin{aligned}
& M=M^{(r)}+M^{(v)}=\sum_{j}\left(M_{j}^{(r)}+M_{j}^{(v)}\right) \\
& M_{j}^{(r)}=\left(k^{4} / 4\right)\left|f_{j}\left(\vec{\alpha}, \vec{\alpha}_{0}\right)\right|^{2} \sigma_{j}^{2} \Phi_{j}^{(r)}(\vec{k}) \\
& M_{j}^{(v)}=\left(k^{4} / 2\right) b_{j}{ }_{j}^{(v)}(\vec{k}) \int_{0}\left|f\left(\vec{\alpha}, \vec{\alpha}_{0}, z+z_{j}\right)\right|^{2} d z
\end{aligned}
$$

where $h_{j}=z_{j+1}-z_{j}$ is the j-layer thickness, $\vec{k}=\vec{\alpha}-\vec{\alpha}_{0}$ is the scattering vector, $\Phi_{j}(v, r)$ are the spatial $2 D$-spectra of the volume inhomogeneities of $j$-layer and the roughness of j-interface, normalized so that $\int_{j}{ }^{(v, r)}(\vec{\kappa}) d^{2} \kappa=1$. For shortening, equations (3) are represented here for the case, when irregularities of different interfaces are uncorrelated and a vertical correlation scale of volume inhomogeneities $b_{j}$ is small comparing with the sound wave length (it is sufficient in practice if $\left.b_{j} k<1\right)$.

The obtained here equations (3) make it possible, if the function $\psi(z, \alpha)$ and consequently $f$ and $f_{j}$ are determined, by specifying spectra $\Phi_{j}^{(v, r)}(\vec{k})$, to evaluate the sound scattering coefficient of an arbitrary layered bottom medium with rough interfaces and volume inhomogeneities.

\section{COMPUTER SIMULATION.}

As an example for computer simulation, we consider the backscattering case, when $\kappa=-2 \alpha_{0}=-2 k \cos \chi$, where $\chi$ is the grazing angle of incident wave near the seabed surface. Besides, the layers are assumed to be homogeneous on the average. Sound absorbtion is taken into account with the advent of refraction index complexity: $n_{j}=n_{j}^{\prime}+$ in $j_{j}$. Spectra $\Phi_{j}{ }^{(v, r)}$ are normalized and specified by power law $\Phi{ }_{j}^{(v, r)}(\vec{k})=a_{j}^{2}\left(\gamma_{j} / 2-1\right) \pi^{-1}\left(1+\kappa^{2} a_{j}^{2}\right)^{-\gamma / 2}$

where $a_{j}=a_{j}{ }^{(v, r)}$ are the horizontal correlation scales of volume inhomogeneities of $j$-layer and roughness of $j$-interface, $\gamma_{j}=\gamma_{j}{ }^{(v, r)}$ are the spectra power indices. 


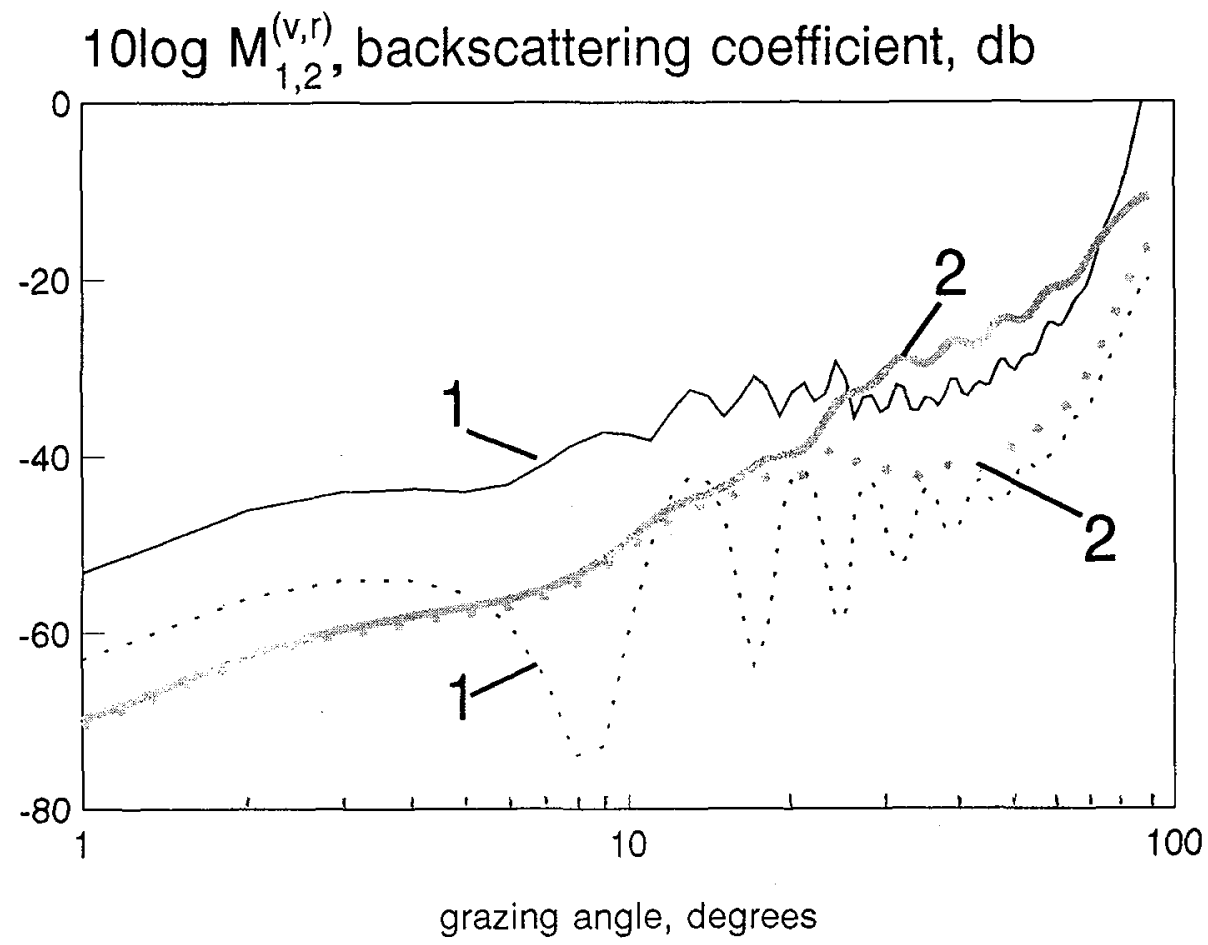

Figure 1. Backscattering coefficients $M_{1,2}{ }^{(v)}$ (solid curves 1,2 , volume scatter) and $M_{1,2}{ }^{(r)}$ (dashed curves 1,2 , roughness scatter) vs grazing angle $\chi$.

At the figure 1, the results of computer simulation are presented for a two layers bottom model $(\mathrm{N}=2)$. The values of the model parameters are listed in the Table 1 and correspond to a silty layer overlaying a sandy half-space.

TABLE 1. Parameters of the bottom model used for the calculations

$\begin{array}{lllllllllllll}\mathrm{j} & \mathrm{m} & \mathrm{n}^{\prime} & \mathrm{n}^{\prime \prime} & \mathrm{kh} & \mathrm{k \sigma} & \mathrm{kb} & \varepsilon & \mu & \mathrm{ka}^{(\mathrm{v})} & \mathrm{ka}^{(\mathrm{r})} & \gamma^{(\mathrm{v})} & \gamma^{(\mathrm{r})} \\ 1 & 1.4 & 1.03 & 0.001 & 30 & 0.1 & 0.3 & 0.01 & 0.1 & 5.0 & 3.0 & 4 & 4 \\ 2 & 2.0 & 0.9 & 0.01 & \infty & 0.1 & 0.3 & 0.03 & 0.1 & 2.0 & 3.0 & 3 & 4\end{array}$

\section{References.}

[1] Ivakin A.N. and Lysanov Yu.P., Sov. Phys. Acoust. 27 (1981) 212-215.

[2] Ivakin A.N., Vopr.Sudostr. Ser. Akust. N17 (1983) 20-25.

[3] Jackson D.R., Winebrenner D.P. and Ishimaru A., J.Acoust.Soc.Am. 79 (1986) 1410-1422.

[4] Jackson D.R. and Briggs K.B., J.Acoust. Soc. Am. 92 (1992) 962-977.

[5] Ivakin A.N., "Sound scattering from ocean bottom: theory and experiment", European Conference on Underwater Acoustics, Luxembourg 14-18 September 1992 (Elsevier Applied Science, 1992) pp.521-524.

[6] Ivakin A.N., Sov. Phys. Acoust. 32 (1986) 492-496.

[7] McDaniel S.T., J.Acoust.Soc. Am. 91 (1992) 1353-1356.

[8] Tang D. and Frisk G.V., J.Acoust. Soc. Am. 92 (1992) 2792-2799.

[9] Hamilton E.L. and Bachman R.T., J.Acoust. Soc. Am. 72 (1982) 1891-1904.

[10] Brekhovskikh L.M. and Godin O.A., Acoustics of layered media (Nauka, Moscow, 1989) pp. 25-89. 